\title{
STUDY ON POST-OPERATIVE INFECTIONS WITH TEICOPLANIN PROPHYLAXIS IN TOTAL KNEE ARTHROPLASTY
}

\author{
B. Narendra Reddy', P. Anil Babu²
}

${ }^{1}$ Associate Professor, Department of Orthopaedics, NRI Medical College, Chinakakani, Guntur, Andhra Pradesh. ${ }^{2}$ Assistant Professor, Department of Orthopaedics, Guntur Medical College, Guntur, Andhra Pradesh.

\section{ABSTRACT}

\section{BACKGROUND}

Teicoplanin is an antibiotic used in the prophylaxis and treatment of serious infections caused by Gram-positive bacteria including methicillin-resistant Staphylococcus aureus and Enterococcus faecalis. It is a glycopeptide antibiotic extracted from Actinoplanes teichomyceticus. Teicoplanin prophylaxis can effectively prevent infections in TKA.

\section{MATERIALS AND METHODS}

The usage of Teicoplanin as prophylaxis in Total Knee Arthroplasty can prevent serious infections post-operatively. This study has been designed as observational study to know the efficacy of prophylaxis for post-operative infections with Teicoplanin in TKA patients. The patients are evaluated for pre- and post-operative infections for local rise of temperature, swelling, pain discharge from the incision site during their follow-ups for three months.

\section{RESULTS}

Out of 1200 cases in this study, there are two post-operative infections. One infection each in the groups of elevated ESR and Positive CRP.

\section{CONCLUSION}

Teicoplanin Prophylaxis is effective in preventing infections in patients undergoing TKA for primary osteoarthritis.

\section{KEYWORDS}

Infection, Teicoplanin Prophylaxis, Total Knee Arthroplasty, Antibiotic.

HOW TO CITE THIS ARTICLE: Reddy BN, Babu PA. Study on post-operative infections with teicoplanin prophylaxis in total knee arthroplasty. J. Evolution Med. Dent. Sci. 2017;6(39):3160-3162, DOI: 10.14260/Jemds/2017/682

\section{BACKGROUND}

Teicoplanin is an antibiotic used in the prophylaxis and treatment of serious infections caused by Gram-positive bacteria including methicillin-resistant Staphylococcus aureus and Enterococcus faecalis. It is a glycopeptide antibiotic extracted from Actinoplanes teichomyceticus with a similar spectrum of activity to vancomycin. Its mechanism of action is to inhibit bacterial cell wall synthesis. Oral teicoplanin has been demonstrated to be effective in the treatment of pseudomembranous colitis and Clostridium difficileassociated diarrhoea with comparable efficacy to vancomycin.

The Staphylococci infections contribute to approximately $70 \%$ of post-operative infections in orthopaedic prosthetic surgery, most commonly with Staphylococcus epidermidis. Therefore, the antibiotics most widely used for prophylaxis are cefazolin, cefamandole and cefuroxime by virtue of their excellent activity against these pathogens. A clean prosthetic surgery like Total Knee Arthroplasty requires antibiotic cover, which should counter methicillin resistant coagulase positive and negative staphylococci. The incidence of these infections is increasingly being reported as the causative agents of postoperative infection in clean prosthetic surgery. ${ }^{1}$

Financial or Other, Competing Interest: None.

Submission 07-04-2017, Peer Review 02-05-2017,

Acceptance 08-05-2017, Published 15-05-2017.

Corresponding Author:

Dr. P. Anil Babu,

\#5-54-120, 101 Suryodaya Residency,

6/18, Brodipet, Guntur-522002,

Andhra Pradesh.

E-mail: anilpbabu@gmail.com

DOI: $10.14260 /$ jemds $/ 2017 / 682$
The systemic changes associated with acute inflammation are collectively called the acute-phase response or the systemic inflammatory response syndrome. These changes are reactions to cytokines whose production is stimulated by bacterial products such as LPS and by other inflammatory stimuli. The acute-phase response consists of several clinical and pathologic changes: Fever, characterised by an elevation of body temperature usually by $1^{\circ}$ to $4^{\circ} \mathrm{C}$, is one of the most prominent manifestations of the acute-phase response, especially when inflammation is associated with infection. Fever is produced in response to pyrogens that act by stimulating prostaglandin synthesis in the vascular and perivascular cells of the hypothalamus. Bacterial products, such as LPS (exogenous pyrogens) stimulate leukocytes to release cytokines such as IL-1 and TNF (called endogenous pyrogens) that increase the enzymes (cyclooxygenases) that convert AA into prostaglandins. In the hypothalamus the prostaglandins, especially PGE2 stimulate the production of neurotransmitters such as cyclic adenosine monophosphate, which function to reset the temperature set point at a higher level.

Acute-phase proteins are plasma proteins, mostly synthesised in the liver, whose plasma concentrations may increase several hundred-fold as part of the response to inflammatory stimuli. Three of the best-known of these proteins are C-Reactive Protein (CRP), fibrinogen and serum amyloid A (SAA) protein. Synthesis of these molecules by hepatocytes is up-regulated by cytokines, especially IL-6 (for CRP and fibrinogen) and IL-1 or TNF (for SAA). Many acute-phase proteins such as CRP and SAA, bind to microbial cell walls and they may act as opsonins and fix complement. They also bind chromatin, possibly aiding in the clearing of 
necrotic cell nuclei. During the acute-phase response SAA protein replaces apolipoprotein A, a component of highdensity lipoprotein particles. This may alter the targeting of high-density lipoproteins from liver cells to macrophages, which can use these particles as a source of energy-producing lipids. Fibrinogen binds to red cells and causes them to form stacks (Rouleaux) that sediment more rapidly at unit gravity than do individual red cells. This is the basis for measuring the erythrocyte sedimentation rate as a simple test for the systemic inflammatory response caused by any stimulus.

Inflammation is present during all stages of any infection and is intimately linked with presentation of an infection. Assessment of systemic inflammation has become important in overall risk stratification, subdued local signs of infection are observed. While a number of circulating markers of inflammation correlate with risk, C-reactive protein (CRP) has emerged as one of the simplest. CRP is an acute-phase reactant synthesised primarily by the liver. It is downstream of a number of inflammatory triggers and plays a role in the innate immune response by opsonising bacteria and activating complement.

Local signs of infection following a surgery include swelling, redness, pain, local rise of temperature, wound dehiscence, discharge from the surgical wound. Presence of any of the local or systemic effects should rise the suspicion of infection. (2)

Infection in orthopaedic surgery is one of the most dreaded complications. It is associated with prolonged morbidity, disability and increased mortality. Surgical site infection in clean wounds (Closed uninfected wounds) include incisional and organ space infections.(3) Teicoplanin with such a spectrum can be used in TKA as prophylaxis, (4) as the dreaded infections are also treated by Teicoplanin.

\section{MATERIALS AND METHODS}

Our study consists of 1200 cases who have undergone unilateral TKA for primary osteoarthritis of knee joint. All the cases are pre-operatively evaluated with surgical profile as a routine for their fitness to undergo surgery as well as to make out presence of indicators of infection including Complete Blood Picture with levels of ESR and C-reactive protein. The study is designed as observational study, the patients for the initial post-operative three months are monitored during their regular followup with inspection of the surgical wounds and body temperature during their hospital stay. After discharge, followup is done for any local signs of infection, viz. swelling, change of skin colour, local rise of temperature or wound dehiscence.

The cases in our study are grouped under two categories, viz. ESR Category and CRP Category. They are again grouped as ESR level below $50 \mathrm{~mm}$ and above $51 \mathrm{~mm}$. The CRP group has CRP Positives and CRP Negatives. With this categorisation, the size of each group has become like cases with ESR $<50$ mm: 639 cases and > $51 \mathrm{~mm}$ : 559. The CRP group has Positives: 339 and Negatives: 859.

All the cases are given a single dose of intravenous Teicoplanin $400 \mathrm{mg}$ one hour prior to Surgery. The surgery under tourniquet control lasted for 51 minutes on an average. Before the wound closure all cases are given a cocktail consisting of Bupivacaine $0.5 \% 5 \mathrm{~mL}$, Tranexamic acid $500 \mathrm{mg}$,
Depomedrol $80 \mathrm{mg}$, Cefuroxime $1 \mathrm{gm}$ into $3 \mathrm{~mm}$ depth of the surgical planes. All of them received 6 doses of Ceftriaxone, post-operatively as BID dose intravenously. The cases are discharged on post-operative day four, by that time two dressing changes are done with monitoring for any local signs of infection. The cases are discharged with no antibiotics. The post-operative followup is spaced as $11-14$ days for suture removal, fourth post-operative week, eighth post-operative week and twelfth post-operative week.

\section{RESULTS}

The dressing changes during the hospital stay of the patients up to fourth post-operative day and surgical site inspection on three postop visits are utilised for monitoring the patients for any local signs of infection like swelling, redness, pain, local rise of temperature, wound dehiscence, discharge from the surgical wound. Presence of any of the local or systemic effects should rise the suspicion of infection. Two cases presented with all the said features between eight and twelfth postoperative week. These two cases are previously categorised into raised ESR level to $>51 \mathrm{~mm}$ and CRP Positive group.

Rest of the 1198 cases showed good wound healing without any swelling, redness, pain, local rise of temperature, wound dehiscence, discharge from the surgical wound. The significance of Teicoplanin prophylaxis is seen as absence of any of the signs of infection in primary osteoarthritis patients undergoing TKA. The results can be analysed as high values of ESR and Positive CRP in the absence of clinical signs of infection are continuously not showing signs of infection perioperatively as well as during post-operative follow-ups. The results can be analysed as one post-operative infection in the cases where ESR is preoperatively elevated and one postoperative infection in the case where CRP is positive.

The elevated ESR group has one case with Chi-square statistic at 0.0089 and $p=0.924656$, both indicating the ESR elevation alone has got no significance. The CRP Positive group has one case with Chi-square statistic at 0.4632 and $\mathrm{p}=$ 0.496541 , both indicating the CRP Positive status elevation alone has got no significance.

\begin{tabular}{|c|c|c|c|}
\hline ESR Level & $<\mathbf{5 0} \mathbf{~ m m}$ & $>\mathbf{5 1} \mathbf{~ m m}$ & Total \\
\hline No. of Cases & 639 & 559 & 1200 \\
\hline Infection & 0 & 1 & 1 \\
\hline \multicolumn{4}{|c|}{ Table 1. Pre-Operative ESR } \\
\hline
\end{tabular}

\begin{tabular}{|c|c|c|c|}
\hline CRP Status & Positive & Negative & Total \\
\hline No. of Cases & 330 & 859 & 1200 \\
\hline Infection & 1 & 0 & 1 \\
\hline \multicolumn{4}{|r|}{ Table 2. Pre-Operative CRP Status } \\
\hline
\end{tabular}

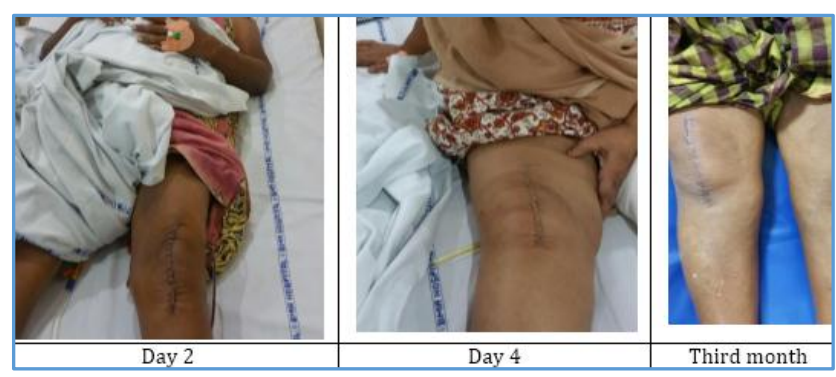

Figure 1. Clean Cases 


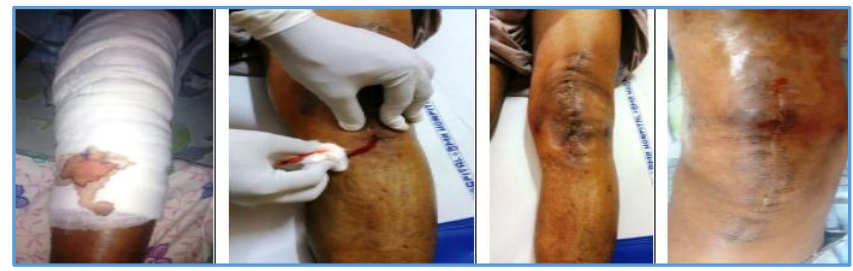

Figure 2. Infected Cases

\section{DISCUSSION}

Infection in an orthopaedic surgery can influence the outcome in an adverse manner and is associated with prolonged morbidity, disability and increased mortality. The World Health Organization (WHO) has recommended items for surgical safety checklist before any surgical procedure to reduce complications. It also includes prophylactic antibiotics. Systemic antibiotic prophylaxis for orthopaedic implant surgeries is the standard practice of care being used for last few decades. Many studies have shown that even with ultraclean theatres, prophylactic antibiotics reduce the risk of infection where an implant is being used, although the evidence is not entirely undisputed. There is enough evidence to say that prophylactic antibiotics should be used in orthopaedics to reduce surgical site infection. The issues to be considered in using prophylactic antibiotics are- (1) Timing of administration, (2) Which antibiotics to be used and (3) Duration of prophylactic antibiotics.

The preoperative antibiotic prophylaxis is crucial to ensure adequate antibiotic concentration. The timing of administration remains controversial. It varies in different studies from 15 mins. to 120 mins. before the skin incision. Yeap et al advocated administration of antibiotics $30-60$ mins. before the surgery or at the time of induction of anaesthesia or at least 10 mins. before inflation of tourniquet. The authors reported that initiating prophylaxis after the skin is incised is ineffective. Administration > 60 mins. before surgery/incision is associated with higher risk of surgical infections. The prophylaxis has least effect when antibiotic is given after the application of a tourniquet. Thus, the extremity remains unprotected for a large duration from antibiotic prophylaxis.

The type of antibiotics administered at any time preoperatively are $\beta$-lactams such as cephalosporins, penicillin and its derivatives such as cloxacillin, glycopeptides as teicoplanin and aminoglycosides such as gentamicin. Thonse et al recommended prophylactic antibiotic regimen at time of induction of anaesthesia and two subsequent doses at 8 and 16 h post-operatively. The duration of post-operative antibiotics is highly variable, and single dose prophylaxis was rarely practiced. Thus, there is still considerable discussion in the current literature as to the duration of prophylactic antibiotics and no consensus has yet arrived.

Teicoplanin as IV infusion into foot veins under a tourniquet block was tried in patients undergoing primary elective monolateral or bilateral Total Knee Replacement (TKR) were consecutively enrolled in a prospective, open clinical study on the efficacy and safety of regional prophylaxis with Teicoplanin (TEC). Those scheduled for monolateral TKR (115 patients) received $400 \mathrm{mg}$ of TEC in $100 \mathrm{~mL}$ of saline as 5 -mins. infusion into a foot vein of the leg to be operated on immediately after the tourniquet was inflated to $400 \mathrm{mmHg}$.

\section{CONCLUSION}

As the results are showing that elevated ESR and positive CRP are not related to the occurrence of post-operative infections. Teicoplanin used as prophylaxis is effectively preventing infections even in the presence of elevated ESR in all the cases and positive status of CRP. Thus, our study concludes that Teicoplanin as prophylaxis prevents post-operative infections irrespective of elevated ESR and Positive CRP status for patients undergoing TKA.

\section{REFERENCES}

[1] De Lalla F. Antibiotic prophylaxis in orthopedic prosthetic surgery. Chemother 2001;13(Suppl 4): Spec No 1:48-53.

[2] Lazzarini L, Pellizzer G, Stecca C, et al. Postoperative infections following total knee replacement: an epidemiological study. J Chemother 2001;13(2):182-7. http://dx.doi.org/10.1179/joc.2001.13.2.182.

[3] Dhammi IK, Ul Haq R, Kumar S. Prophylactic antibiotics in orthopedic surgery: Controversial issues in its use: Indian J Orthop 2015;49(4):373-6.

[4] de Lalla F, Novelli A, Pellizzer G, et al. Regional and systemic prophylaxis with teicoplanin in monolateral and bilateral total knee replacement procedures: study of pharmacokinetics and tissue penetration. Antimicrob Agents Chemother 1993;37(12):2693-8. 